\title{
Opinion \\ What health professionals say equals gold
}

Volume 4 Issue 5 - 2015

\section{Opinion}

The problem of health care costs have always been an issue discussed by decisions-makers and politicians. We always focus on how much we spend on medication and treatments and how it could be possible to spend less via using less expensive medicines and optimize therapeutic approaches, in addition of dealing with the drugs markets. $^{1}$

A better approach could be to work at the basic level which is represented by the interactions and the communications between the health care professionals and the patients (and their families). Indeed, a better education in term of diet, exercise, sleeping cycle ${ }^{2}$ and psychological health could considerably reduce the health care expenses. For instance, the impacts prevention has on the health of the general population is well known therefore, populations who have a deep understanding of the prevention have a reduced risk of developing health problems thus, a lower cost of the health care.

Following this line of thoughts, the concept of education could also be applied during the treatment (in addition to the prevention). Illustrative examples could be represented using the medicines in a rational way, use of health products, selecting food, cooking ways and the use of the traditional medicines. ${ }^{3,4}$ Such concepts will also have a strong impact on the budgets of the health care. Furthermore, regulations and legislations, ${ }^{5}$ in addition of training the health care professionals $\mathrm{s}^{6,7}$ will also allow further reduce and control how much a country will spend on health care.

\section{Acknowledgements}

Abdelaziz Ghanemi is a recipient of a 2013 CAS-TWAS President's Postgraduate Fellowship.

\begin{abstract}
Abdelaziz Ghanemi ${ }^{1,2,3}$
'Key Laboratory of Animal Models and Human Disease Mechanisms of the Chinese Academy of Sciences \& Yunnan Province, Kunming Institute of Zoology, Chinese Academy of Sciences , Kunming, Yunnan 650223, PR China ${ }^{2}$ Kunming College of Life Science, University of Chinese Academy of Sciences, Kunming 650204, China

${ }^{3}$ University of Chinese Academy of Sciences, Beijing I0049, PR China

Correspondence: Abdelaziz Ghanemi, Key Laboratory of Animal Models and Human Disease Mechanisms, Kunming Institute of Zoology Chinese Academy of Sciences, No. 32 Jiaochang Donglu, Kunming 650223, China, Email ghanemiabdelaziz@hotmail.com
\end{abstract}

Received: December 21, 2015 | Published: December 22, 2015

\section{Conflicts of interest}

Author declatres there are no conflicts of interest.

\section{Funding}

None.

\section{References}

1. Tratados Hipocráticos. Biblioteca Clásica Gredos. 1983;63. 